Retch

\title{
Defects of the Carney complex gene (PRKAR1A) in odontogenic tumors
}

\author{
Sílvia F Sousa', Ricardo S Gomez', Marina G Diniz', Vanessa F Bernardes², \\ Flávia F C Soares ${ }^{1}$, João Artur R Brito ${ }^{1}$, Sophie Liu ${ }^{3}$, Hélder Antônio R Pontes ${ }^{4}$, \\ Constantine A Stratakis ${ }^{3}$ and Carolina C Gomes ${ }^{2}$
}

${ }^{1}$ Department of Oral Surgery and Pathology, School of Dentistry, and ${ }^{2}$ Department of Pathology, Biological Sciences Institute, Universidade Federal de Minas Gerais (UFMG), Avenida Antônio Carlos, 6627, Belo Horizonte, Minas Gerais CEP 31270-901, Brazil

${ }^{3}$ Section on Endocrinology and Genetics, Program on Developmental Endocrinology and Genetics (PDEGEN), NIH, Bethesda, Maryland, USA

${ }^{4}$ João de Barros Barreto University Hospital, Universidade Federal do Pará (UFPA), Belém, Brazil

Correspondence should be addressed to C C Gomes Email carolinacgomes@ufmg.br

\begin{abstract}
The surgical treatment of some odontogenic tumors often leads to tooth and maxillary bone loss as well as to facial deformity. Therefore, the identification of genes involved in the pathogenesis of odontogenic tumors may result in alternative molecular therapies. The PRKAR1A gene displays a loss of protein expression as well as somatic mutations in odontogenic myxomas, an odontogenic ectomesenchymal neoplasm. We used a combination of quantitative RT-PCR (qRT-PCR), immunohistochemistry, loss of heterozygosity (LOH) analysis, and direct sequencing of all PRKAR1A exons to assess if this gene is altered in mixed odontogenic tumors. Thirteen tumors were included in the study: six ameloblastic fibromas, four ameloblastic fibro-odontomas, one ameloblastic fibrodentinoma, and two ameloblastic fibrosarcomas. The epithelial components of the tumors were separated from the mesenchymal by laser microdissection in most of the cases. We also searched for odontogenic pathology in Prkar1a ${ }^{+/-}$mice. PRKAR1A mRNA/protein expression was decreased in the benign mixed odontogenic tumors in association with $\mathrm{LOH}$ at markers around the PRKAR1A gene. We also detected a missense and two synonymous mutations along with two $5^{\prime}$-UTR and four intronic mutations in mixed odontogenic tumors. Prkar1a ${ }^{+/-}$mice did not show evidence of odontogenic tumor formation, which indicates that additional genes may be involved in the pathogenesis of such tumors, at least in rodents. We conclude that the PRKAR1A gene and its locus are altered in mixed odontogenic tumors. PRKAR1A expression is decreased in a subset of tumors but not in all, and Prkar1a ${ }^{+1-}$ mice do not show abnormalities, which indicates that additional genes play a role in this tumor's pathogenesis.
\end{abstract}

\section{Key Words}

- mixed odontogenic tumors

- ameloblastic fibroma

- ameloblastic fibro-odontoma

- ameloblastic fibrodentinoma

- ameloblastic fibrosarcoma

- loss of heterozygosity

- benign tumors

\section{Introduction}

Odontogenic tumors arise from odontogenic tissues, and they can be aggressive and destructive lesions (Barnes et al. 2005). Surgical treatment may result in high morbidity, because it can lead to tooth and maxillary bone loss as well as facial deformity (Praetorius 2009). Therefore, elucidation of the molecular pathogenesis of these lesions may be useful in designing targeted therapies to be used in large, destructive, aggressive, or recurrent cases.

Published by Bioscientifica Ltd.
Endocrine-Related Cancer (2015) 22, 399-408 
Mixed odontogenic tumors are a group of rare tumors that have been classified by the World Health Organization (WHO) as odontogenic tumors with odontogenic epithelium and odontogenic ectomesenchyme with or without hard tissue formation (Barnes et al. 2005). This group of tumors comprises the ameloblastic fibroma (AF), ameloblastic fibro-odontoma (AFO), ameloblastic fibrodentinoma (AFD), odontoameloblastoma, deninogenic ghost-cell tumor, calcifying cystic odontogenic tumor, and the odontomas.

The AF is a rare benign tumor characterized by a proliferation of odontogenic ectomesenchyme that resemble the dental papilla, with epithelial strands and nests similar to the dental lamina and enamel organ (Barnes et al. 2005). It is regarded as a true neoplastic lesion with a risk of malignant transformation of the mesenchymal counterpart into ameloblastic fibrosarcoma (AFS) (Philipsen et al. 1997, Bernardes et al. 2012). In previous studies that included Brazilian as well as Mexican cases, AF represented about $0.7 \%$ of the odontogenic tumors and AFS represented only $0.13 \%$ (Galvão et al. 2012). In the presence of hard tissues such as dentin or enamel, the lesion may be classified as AFD or AFO respectively (Barnes et al. 2005). $\mathrm{AF}, \mathrm{AFD}$, and $\mathrm{AFO}$ belong to the group of mixed odontogenic tumors. These lesions histopathologically resemble various stages of tooth formation, although there is still debate about whether they are separate entities or different stages in the maturation of the same lesion (Praetorius 2009).

Several studies have focused on the molecular pathogenesis of the odontogenic tumors; however, because mixed odontogenic tumors are rare, studies involving their pathogenesis are scarce. Genes involved in the process of odontogenesis and tumor suppressor genes have been the main targets of molecular studies of odontogenic tumors (Barreto et al. 2000, Perdigão et al. 2004, Gomes \& Gomez 2011, Farias et al. 2012). In this context, our group described loss of heterozygosity (LOH) at tumor suppressor gene loci in mixed odontogenic AF and AFO tumors as well as in AFS (Galvão et al. 2012).

PRKAR1A is a tumor-suppressor gene that encodes protein kinase A (PKA) regulatory subunit $1 \alpha$. A reduction in the cellular levels of R1 $\alpha$ results in increased PKA/cAMP signaling and activation of the processes of proliferation and differentiation (Robinson-White et al. 2006). This gene is mutated in sporadic (Maleszewski et al. 2014) and in syndromic myxomas associated with Carney complex (Kirschner et al. 2000a). On the basis of histological similarities between the cardiac myxomas and the odontogenic myxomas, our group assessed PRKAR1A gene expression in odontogenic myxomas and described decreased protein expression as well as the presence of one missense mutation and a single deletion (Perdigão et al. 2005). Although dental myxomas have not been described in patients with Carney complex, this association should be known to clinicians who are caring for patients with the complex.

We recently assessed PRKAR1A and PRKAR2A mRNA expression by quantitative RT-PCR (qRT-PCR) and in situ hybridization during normal human and murine odontogenesis (de Sousa et al. 2014). Considering PRKAR1A expression in normal tooth development (de Sousa et al. 2014), and its alterations in the ectomesenchyme of odontogenic myxomas (Perdigão et al. 2005), we proposed the hypothesis that such molecular alterations might be shared by other odontogenic tumors that contain an ectomensechymal component (AF, AFO, AFD, and AFS). In the present study, we aimed to investigate molecular alterations of PRKAR1A in a set of mixed odontogenic tumors by using qRT-PCR, immunohistochemistry (IHC), $\mathrm{LOH}$ analysis, and direct sequencing. We further investigated the occurrence of odontogenic tumors in Prkar1a ${ }^{+/-}$mice, because facial bones had never before been specifically analyzed in these animal models (Kirschner et al. 2005).

\section{Materials and methods}

\section{Odontogenic tumors: identification and recruitment}

The present study was approved by the Ethics Committee of the Universidade Federal de Minas Gerais (protocol no. 498/11). Fresh tissue samples were collected from patients who attended at Oral Diagnosis Service at the Dentistry School of UFMG after they provided written informed consent to participate in the present study. Twenty fresh samples were included in the qRT-PCR, consisting of seven ameloblastomas (AM) as a comparison group, one AF (case no. 1), one AFD (case no. 9), five normal oral mucosa $(\mathrm{OM})$, and six pericoronal follicles. Pericoronal follicles were obtained from impacted third molars free of clinical inflammation, and the normal $\mathrm{OM}$ included were obtained during surgeries on odontogenic tumors.

Because of the rarity of mixed odontogenic tumors, we included an additional 11 formalin-fixed paraffinembedded (FFPE) samples: five AF, four AFO and two odontogenic sarcomas (AFS). Table 1 shows the odontogenic tumors investigated and the experiments performed with each tumor.

Published by Bioscientifica Ltd. 
Table 1 Demographic data for the odontogenic tumors included and the experiments carried out on each of them

\begin{tabular}{|c|c|c|c|c|c|c|c|}
\hline \multirow[b]{2}{*}{ Sample } & \multirow[b]{2}{*}{ Sex } & \multirow[b]{2}{*}{ Age (years) } & \multirow[b]{2}{*}{ Location } & \multicolumn{4}{|c|}{ Assay } \\
\hline & & & & $\mathrm{IHC}$ & MD & $\mathrm{LOH}$ & SEQ \\
\hline \multicolumn{8}{|c|}{ Ameloblastic fibroma } \\
\hline Case $1^{\mathrm{a}}$ & $\mathrm{F}$ & 7 & Mandible & $\checkmark$ & LCM & $\checkmark$ & $\checkmark$ \\
\hline Case 2 & $\mathrm{M}$ & 14 & Mandible & - & $\mathrm{MM}$ & $\checkmark$ & $\checkmark$ \\
\hline Case 3 & $\mathrm{M}$ & 13 & Mandible & - & LCM & $\checkmark$ & - \\
\hline Case 4 & $\mathrm{~F}$ & 6 & Mandible & $\checkmark$ & LCM & $\checkmark$ & $\checkmark$ \\
\hline Case 5 & $\mathrm{~F}$ & NA & NA & $\checkmark$ & MM & $\checkmark$ & $\checkmark$ \\
\hline Case 6 & $\mathrm{~F}$ & 23 & Mandible & $\checkmark$ & - & - & $\checkmark$ \\
\hline \multicolumn{8}{|c|}{ Ameloblastic fibrosarcoma } \\
\hline Case 7 & $\mathrm{M}$ & 25 & Mandible & $\checkmark$ & LCM & $\checkmark$ & $\checkmark$ \\
\hline Case 8 & $\mathrm{~F}$ & 32 & Mandible & $\checkmark$ & LCM & $\checkmark$ & - \\
\hline \multicolumn{8}{|c|}{ Ameloblastic fibrodentinoma } \\
\hline Case $9^{a}$ & $\mathrm{M}$ & 9 & Maxilla & $\checkmark$ & LCM & $\checkmark$ & $\checkmark$ \\
\hline \multicolumn{8}{|c|}{ Ameloblastic fibro-odontoma } \\
\hline Case 10 & $\mathrm{M}$ & 9 & Mandible & $\checkmark$ & MM & $\checkmark$ & $\checkmark$ \\
\hline Case 11 & $\mathrm{M}$ & 6 & Mandible & $\checkmark$ & LCM & $\checkmark$ & $\checkmark$ \\
\hline Case 12 & $\mathrm{~F}$ & 13 & Mandible & $\checkmark$ & - & - & - \\
\hline Case 13 & $\mathrm{~F}$ & 12 & Mandible & $\checkmark$ & - & - & - \\
\hline
\end{tabular}

F, female; M, male; NA, not available; MD, microdissection; LCM, laser capture microdissection; MM, manual microdissection; LOH, loss of heterozygosity; IHC, immunohistochemistry; SEQ, sequencing; $\checkmark$, performed; -, could not be performed.

${ }^{a}$ Cases 1 and 9 were fresh tumors. Case 6 did not have a capsule for the evaluation of normal tissue.

All of the tumors had hematoxylin and eosin (HE) slides revised by at least two pathologists. Diagnoses were based on previously reported criteria (Barnes et al. 2005).

\section{Tissue processing}

All of the samples had a provisional diagnosis based on an incisional biopsy, but final diagnosis was based on an excisional biopsy, which entirely represents the tumor. Tumors were collected during the excisional biopsies. A portion of each fresh tissue collected was stored in an RNA holder (Bioagency Biotecnologia, São Paulo, SP, Brazil) and in tissue-tek (Sakura Finetek, Torrance, CA, USA). RNA holder samples were stored at $4{ }^{\circ} \mathrm{C}$ for $24 \mathrm{~h}$ before being frozen in liquid nitrogen, and tissue-tek samples were immediately immersed in nitrogen. A third portion was FFPE for diagnosis confirmation.

\section{RNA extraction and qRT-PCR}

Total RNA was extracted from the fresh samples of AM $(n=7)$, AF $(n=1)$, AFD $(n=1)$, normal OM $(n=5)$, and pericoronal follicles $(n=6)$ using the TRIZOL reagent method (Invitrogen Life Technologies), and it was evaluated by comparative threshold cycle $(C t)$ qRT-PCR. After careful evaluation, a pool of six dental follicles were chosen as calibrators because they homogenously expressed the target genes and the endogenous gene,
$28 \mathrm{~S}$ rRNA. The target genes were PRKAR1A (F: 5'-TCTGTGTGCAACTAACTGACTCTGTT-3' and R: 5'-CCTCTTCCCCAGATTTATACTTCATACT-3'; amplicon size $145 \mathrm{bp}$ ) and PRKAR2A (F: 5'-GGCCCAACCCGTCTATCC-3' and R: 5'-GCAGCACCTCCACCGTGTAG-3'; amplicon size $102 \mathrm{bp}$ ). The average $C t$ for three replicates per sample was used to calculate $\Delta C$ t. The relative quantification of these gene expressions was calculated using the $2^{-\Delta \Delta C t}$ formula (Livak \& Schmittgen 2001). A melting curve analysis followed each reaction to ensure specificity. qRT-PCR was carried out using $1 \times$ SYBR Green PCR Master Mix (Applied Biosystems), as described previously by de Sousa et al. (2014). Expression in odontogenic tumors were compared with that in a normal OM control group.

\section{Immunohistochemistry}

On the basis of the decreased transcriptional levels of PRKAR1A in the set of odontogenic tumors evaluated, we investigated the immunohistochemical expression of the protein in a larger number of samples, including seven epithelial (AMs) and 11 mixed odontogenic tumors. IHC was not carried out in case nos 2 and 3 because of the small amount of available tissue. Tissue sections of $4 \mu \mathrm{m}$ thickness were mounted for IHC on StarFrost knittel glass adhesive slides (Waldemar Knittel Glasbearbeitungs GmbH, Braunschweig, Germany). Immunohistochemical reactions

Published by Bioscientifica Ltd 
were performed using a mouse MAB against the PRKAR1A protein (clone 6C7) (OriGene, Rockville, MD, USA). The processes of deparaffinization, hydration, and antigen retrieval were performed by immersing the slides in Trilogy solution (Cell Marque, Rocklin, CA, USA) and heating them to $98^{\circ} \mathrm{C}$ in a steamer for $20 \mathrm{~min}$. The primary antibody was diluted at 1:80 and incubated for $30 \mathrm{~min}$ at room temperature. EnVision+Dual Link System-HRP (Dako, Carpinteria, CA, USA) was used as the detection system for $30 \mathrm{~min}$ at room temperature. The reactions were revealed by applying 3,3'-diaminobenzidine in chromogen solution (DAB; Dako), and Mayer's hematoxylin was used for counterstaining. The slides were mounted in Permount (Fisher Scientific, Fair Lawn, NJ, USA). IHC was performed manually, and normal $\mathrm{OM}$ was used as positive control. Negative controls were carried out by omission of the primary antibody.

The IHC results were analyzed by three investigators (R S G, S F S, and V F B), who considered the distribution of antibody staining in the entire lesion section, regardless of staining intensity, and categorized the staining as: strong, in the presence of evident staining in the majority of tumor cells (>50\%); moderate, when a significant proportion of the neoplastic cells were positive (10-50\%); and weak/negative, in the absence of marked cells or the presence of only occasional stained cells $(<10 \%)$.

\section{Microdissection}

Manual microdissection (MM) and laser capture microdissection (LCM) were performed on all 11 FFPE samples of the mixed odontogenic tumors that harbored normal and tumor areas (Table 1). For MM, an H\&E slide was superimposed against a paraffin block for delimitation of the tumor from the capsule. Ten sections were cut at $10 \mu \mathrm{m}$ thickness for posterior DNA extraction. For LCM, sections were cut at $12 \mu \mathrm{m}$ thickness onto a MMI membrane (Olympus), and air-dried. Staining procedures were executed as described previously (Strokes et al. 2011). Stained sections were microdissected using an Olympus IX81 microscope and MMI CellCut Plus (Olympus) laser microdissection system. Tumor epithelial cells, tumor mesenchymal cells, and normal cells (capsule or blood vessel endothelium) were collected into separate sterile cap plastic tubes.

\section{DNA isolation}

Microdissected tissue fragments were digested with proteinase K. Genomic DNA from fresh and FFPE samples were extracted using a DNeasy Blood and Tissue Kit (Qiagen) and a QiaAmp DNA FFPE tissue kit (Qiagen) respectively.
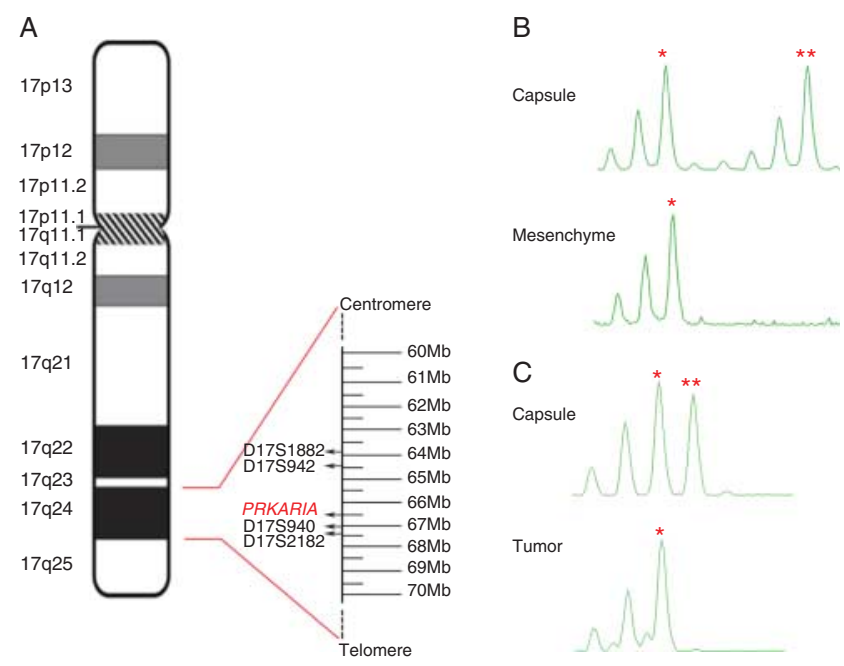

Figure 1

Ideogram of the cytogenetic bands of chromosome 17 and polymorphic microsatellite markers and representative results of PRKAR1A loss of heterozygosity (LOH). (A) Polymorphic DNA markers located on the long arm of chromosome 17, including D17S1882 (17q24.1), D17S942 (17q24.2), D17S940 (17q24.2), and D17S2182 (17q24.3), which are used to assess LOH at the PRKAR1A genetic locus. ( $B$ and $C$ ) Loss of the long allele at marker D17S1882 in the mesenchyme of case 3 (B) and in the tumor of case 5 (C) as compared with matched normal DNA. *short allele, **long allele. A full colour version of this figure is available at http://dx.doi.org/10.1530/ ERC-15-0094.

\section{LOH analysis}

LOH was assessed by using a panel of four polymorphic DNA markers located around PRKAR1A (Fig. 1A). The primers used were selected on the basis of data from the online database (www.ncbi.nlm.nih.gov/probe), and they have been previously used by others (Bertherat et al. 2003). PCR for the four markers was performed as follows: 45 cycles at $96^{\circ} \mathrm{C}$ for $10 \mathrm{~s}, 55^{\circ} \mathrm{C}$ for $30 \mathrm{~s}$, and $70{ }^{\circ} \mathrm{C}$ for $1 \mathrm{~min}$. The PCR products were resolved on $6.5 \%$ nondenaturing polyacrylamide gels with silver staining. All of the PCRs were performed for tumor (epithelium and mesenchyme) and normal (capsule) tissue under the same conditions. Amplification products were detected by capillary electrophoresis on an ABI PRISM 310 (Applied Biosystems). LOH was calculated as previously described by Farias et al. (2012).

\section{Direct sequencing}

DNA targets were PCR-amplified using primer pairs flanking exons $1-10$, as previously described (Kirschner et al. 2000b). Amplified products were confirmed by electrophoresis on $6.5 \%$ polyacrylamide gels with silver

Published by Bioscientifica Ltd 


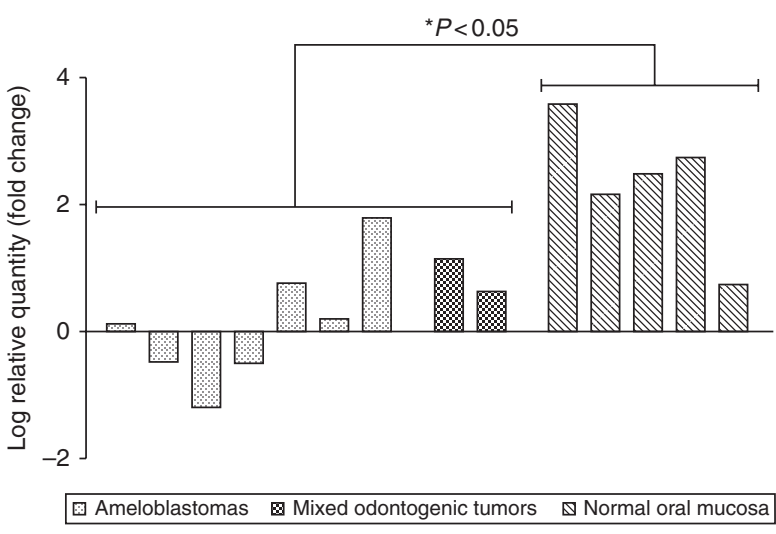

Figure 2

Transcriptional levels of PRKAR1A mRNA analyzed by qRT-PCR in the samples of epithelial (ameloblastoma) and mixed odontogenic tumors as compared to normal OM. PRKAR1A transcription levels in the odontogenic tumors were decreased in relation to normal OM $(P<0.05$, Mann-Whitney $U$ test). The zero level is based on the results for the pool of dental follicles used as reaction calibrator.

staining. PCR products were purified with GFX PCR DNA and a Gel Band Purification Kit (GE Healthcare, Buckinghamshire, UK) and sequenced using a BigDye Terminator (Applied Biosystems) on an ABI PRISM 3130 Genetic Analyzer (Applied Biosystems). Electropherograms were manually analyzed with Sequence Scanner 2 software (Applied Biosystems). The reference sequence adopted was NM_002734.4. All mutation and sequence variances that were found were checked against the Catalogue of Somatic Mutations in Cancer (COSMIC), Ensembl Genome Browser, and NCBI dbSNP databases.

\section{Prkar1a $^{+/-}$mice}

Prkar1 $a^{+/-}$mice (C57BL/6 background) carrying a deletion of exon 2 (Kirschner et al. 2005) were studied. All animal work in the present study was carried out in accordance with the guidelines of the Institutional
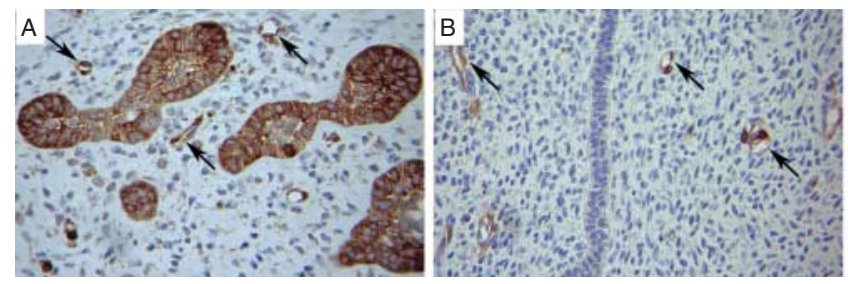

\section{Figure 3}

Immunostaining pattern of PRKAR1A in mixed odontogenic tumors. (A and B) AF. (C) AFD. (D) AFO. Strong expression in the epithelium was observed in (A), (C), and (D). Negative/weak expression in the mesenchyme was observed in (A), (B), and (D). One sample of AF also exhibited an
Laboratory Animal Care and Use Committee at the National Institute of Child Health and Human Development (NICHD), National Institutes of Health (NIH), Bethesda, MD, USA. A total of five 1-month-old mice and four 6-month-old mice were dissected; their orofacial structures were studied after all of the skin had been removed and they had been fixed in 4\% paraformaldehyde (PFA). Bony structures were slowly decalcified with EDTA $0.9 \mathrm{M}$ at $\mathrm{pH} 7.5$ over a period of 20 days, rinsed with distilled water, sectioned, paraffin embedded, and H\&E stained. The sections were analyzed under a light microscope.

\section{Statistical analysis}

Analyses were performed using SPSS version 17.0 (SPSS, Inc.). Normal distribution was assessed by the Shapiro-Wilk test. A Mann-Whitney $U$ test was used to compare the relative quantification of PRKAR1A and PRKAR2A in odontogenic tumors and normal OM. Probability values of $<0.05$ were considered to be statistically significant.

\section{Results}

The results of the molecular analysis (mRNA transcription, IHC, LOH, and sequencing) are presented in Figs 1, 2, 3, 4 and 5. A brief outline of the results is given in the following sections.

\section{Quantitative RT-PCR}

We assessed the mRNA expression levels of PRKAR1A and PRKAR2A in an epithelial odontogenic tumor (AMs, $n=7$ ) and in two samples of fresh mixed odontogenic tumors (one AF and one AFD) and compared these expressions with a control group of five normal OM samples by using
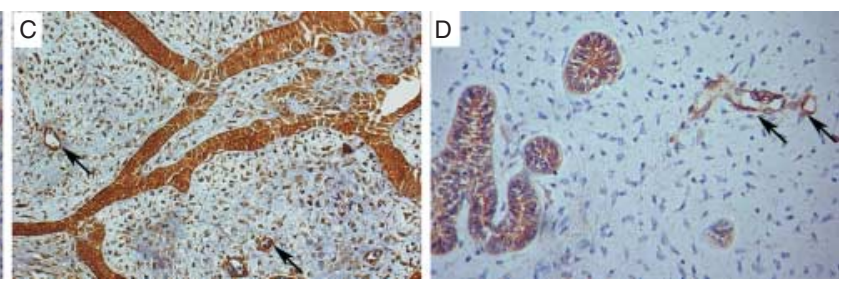

absence of PRKAR1A expression in the epithelium (B). Arrows indicate vessels as internal positive control. Original magnification: $\times 400$ and $\times 100$. A full colour version of this figure is available at http://dx.doi.org/ 10.1530/ERC-15-0094.

Published by Bioscientifica Ltd. 


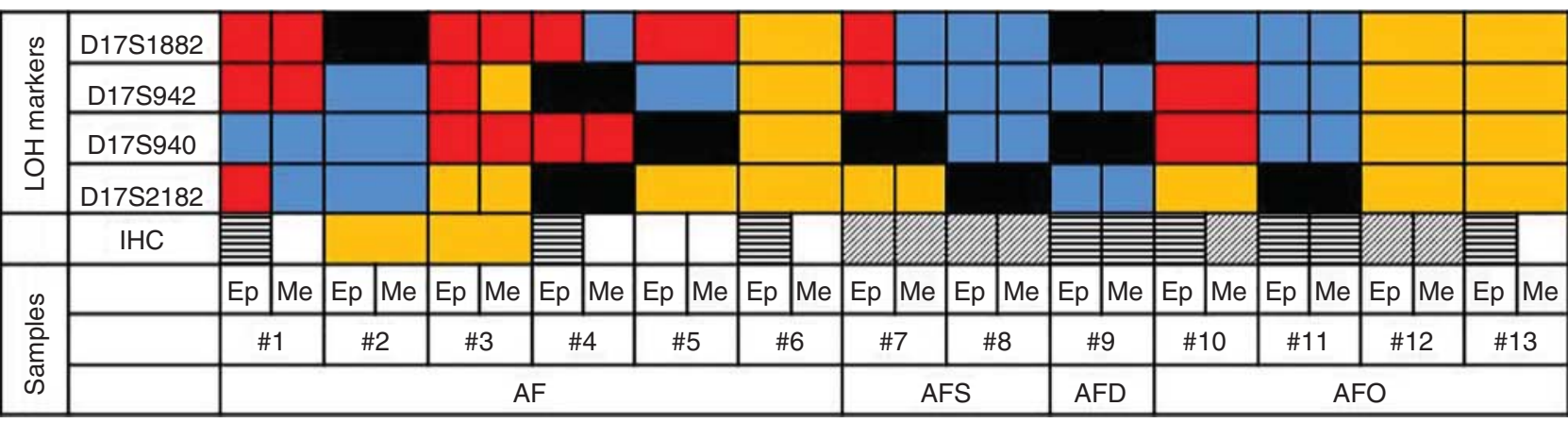

LOH; heterozygous;

homozygous;

not possible to analyze

strong immunoexpression;

moderate immunoexpression;

weak/negative immunoexpression

\section{Figure 4}

Results of the immunohistochemistry and loss of heterozygosity analysis by sample. LOH, loss of heterozygosity; IHC, immunohistochemistry; $E p$, epithelium; Me, mesenchyme; $A F$, ameloblastic fibroma; AFS, ameloblastic fibrosarcoma; AFD, ameloblastic fibrodentinoma; AFO,

a pool of normal dental follicles as reaction calibrators. Both genes were expressed in all of the odontogenic tumors that were evaluated, but the relative quantification of PRKAR1A in odontogenic tumors showed decreased expression as compared with normal OM $(P<0.05)$ (Fig. 2), whereas PRKAR2A mRNA expression was similar between the tumors and normal mucosa (not shown).

\section{Immunohistochemistry}

Although all of the AMs showed positive expression of the protein in the epithelial islands, four AFs showed weak/ negative staining in the ectomesenchymal component (Fig. 3A and B), and one case exhibited negative staining not only in the ectomesenchyme but also in the epithelium (Fig. 3B). The AFD sample showed strong expression in both components (Fig. 3C). Two samples of AFO showed a loss of protein expression in the ectomesenchymal component (Fig. 3D). Blood vessels showed positive staining, which was considered a positive internal control (Fig. 3A, B, C and D). The results are shown in Fig 4.

\section{LOH analysis}

Because the mixed odontogenic tumors exhibited a significant loss of PRKAR1A expression, we investigated if $\mathrm{LOH}$ at the PRKAR1A locus could be one of the underlying mechanisms of decreased expression. We assessed $\mathrm{LOH}$ with a panel of four polymorphic ameloblastic fibro-odontoma. Epithelial and mesenchymal tumor cells could not be separated by laser capture microdissection in cases 2,5 , and 10. A full colour version of this figure is available at http://dx.doi.org/10. 1530/ERC-15-0094.

microsatellite markers (Fig. 1A) in a set of five $\mathrm{AF}$, two AFS, one AFD, and two AFO. For cases 6, 12, and 13, LOH analysis was not carried out either because those cases did not harbor normal control tissue or because the amount of available tissue was small. Four out of five AF exhibited $\mathrm{LOH}$ for at least two of the markers evaluated and presented LOH in the ectomesenchyme and/or epithelium (Fig. 1B). One of the two AFO showed LOH. One AFS exhibited LOH in the epithelium for the same markers that showed LOH in the AF. Only four of the cases investigated (cases 2, 8, 9, and 11) did not have LOH (Fig 4).

\section{Direct sequencing}

Because the PRKAR1A gene is mutated in several human tumors, including the odontogenic myxomas (Perdigão et al. 2005), we sequenced DNA from exons 1-10 in the epithelium and ectomesenchyme of those tumors that had sufficient tissue available (cases 1-2, 4-7, and 9-11). Nucleotide numbering reflects cDNA numbering, with +1 corresponding to the A of the ATG translation initiation codon in the reference sequence (NM_002734.4). All the PRKAR1A mutations and the single nucleotide polymorphisms (SNPs) identified are summarized in Table 2.

We found three sequence alterations in the coding region of the PRKAR1A gene, including two synonymous and one missense alteration. The missense mutation (c.802G $>$ A) was found in exon 8 and only in the

Published by Bioscientifica Ltd 
A

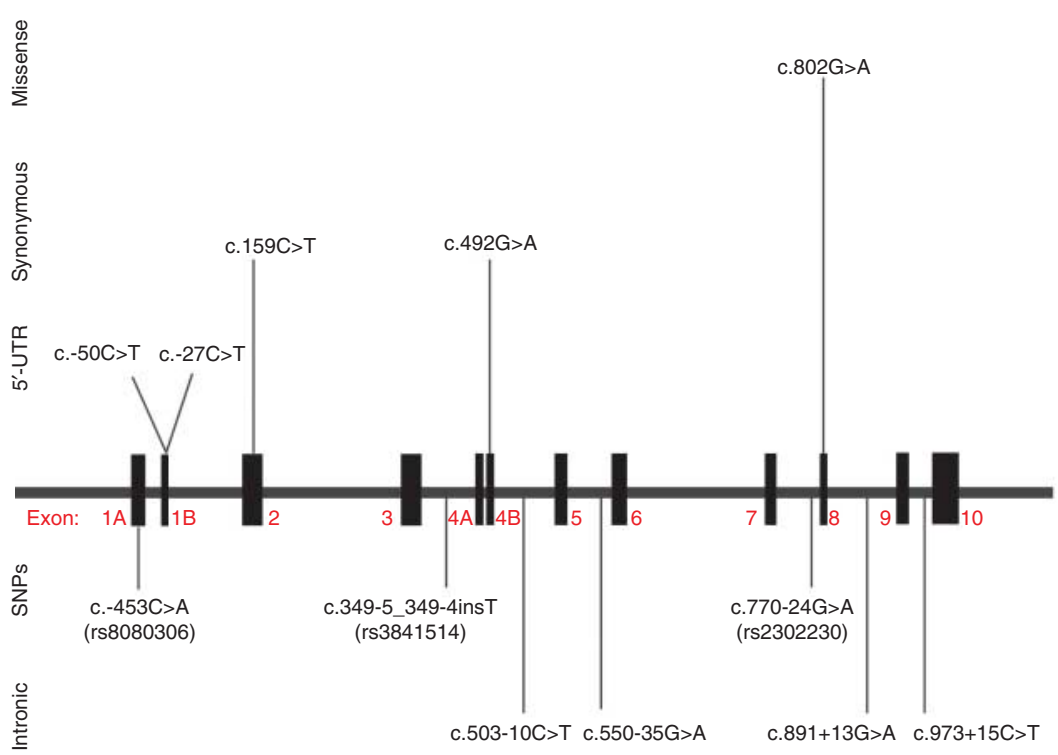

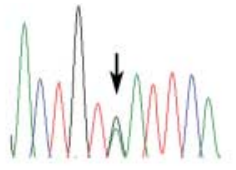

WT: ACT $\underline{G T G A T C A}$

C

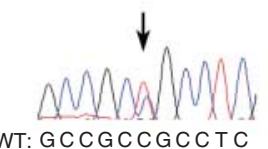

D

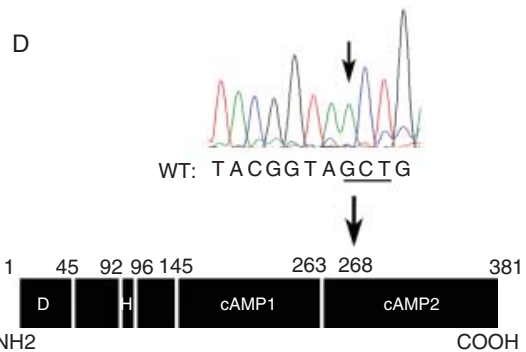

Figure 5

Structure of the PRKAR1A gene and protein, and screenshots of sequencing electropherograms. (A) The locations of all of the sequence alterations and SNPs found in our samples. (B) A heterozygous synonymous sequence change in exon $4 \mathrm{~B}$ (c.492G $>A$ ) (WT codon GTG $\rightarrow$ new codon GTA). (C) A $5^{\prime}$-UTR mutation in exon $1 B$ (c.-50C $>$ T). (D) A missense mutation found in exon 8 (c.802G $>$ A) (WT codon GCT $\rightarrow$ mutant codon ACT) and

ectomesenchymal component of case 11 . This mutation leads to a nonconservative amino acid substitution (p.A268T). The possible effects of this substitution on the stability and function of the protein were predicted to be damaging by SIFT (http://sift.jcvi.org) (score 0.01) and by PolyPhen-2 (http://genetics.bwh.harvard.edu/pph2/) (score 1.000). localization of this mutation in the structure of the PRKAR1A protein. $\mathrm{NH} 2$, amino-terminal extremity; $\mathrm{COOH}$, carboxy-terminal extremity; $D$, dimerization domain; $\mathrm{H}$, hinge region; CAMP1 and CAMP2, CAMP-binding domains. A full colour version of this figure is available at http://dx.doi.org/10.1530/ERC-15-0094.

In addition, we identified two sequence alterations in the $5^{\prime}$-UTR and four intronic variants. We also found three previously identified SNPs. All of the alterations found are represented in Fig. 5A, and the screenshots from the sequencing electropherograms of the missense mutation, one synonymous and one $5^{\prime}$-UTR mutation, are shown in Fig. 5B, C and D.

Table 2 Sequence alterations, including known SNPs of the PRKAR1A gene identified in mixed odontogenic tumors

\begin{tabular}{|c|c|c|c|c|c|}
\hline Mutation & Location & Effect & Diagnosis & Case number & Component \\
\hline C. $-50 \mathrm{C}>\mathrm{T}$ & Exon 1B & $5^{\prime}-U T R$ & $\mathrm{AF}$ & 1 & Epithelial \\
\hline C. $-27 \mathrm{C}>\mathrm{T}$ & Exon 1B & $5^{\prime}$-UTR & $\mathrm{AF}$ & 1 & Epithelial \\
\hline c. $159 C>T$ & Exon 2 & Synonymous & AFD & 9 & Epithelial \\
\hline c. $492 \mathrm{G}>\mathrm{A}$ & Exon 4B & Synonymous & $\mathrm{AF}$ & 5 & Tumor ${ }^{a}$ \\
\hline c. $802 \mathrm{G}>\mathrm{A}$ & Exon 8 & Missense & AFO & 11 & Mesenchymal \\
\hline c. $503-10 C>T$ & Intron 4B-5 & Intronic & AFO & 11 & Epithelial \\
\hline c. $550-35 G>A$ & Intron 5-6 & Intronic & AFO & 11 & Mesenchymal \\
\hline$c .891+13 G>A$ & Intron 8-9 & Intronic & AFO & 11 & Mesenchymal \\
\hline c. $973+15 C>T$ & Intron 9-10 & Intronic & AFO & 11 & Mesenchymal \\
\hline \multicolumn{6}{|l|}{ SNPs } \\
\hline c. $-453 C>A$ & Exon 1A & $5^{\prime}$ UTR & $A F, A F O$ & 1,11 & Both \\
\hline c.349-5_349insT & Intron 3-4A & Intronic & $A F, A F D, A F O$ & $1,9,11$ & Both \\
\hline c. $770-24 G>A$ & Intron 7-8 & Intronic & $A F, A F D, A F O$ & $1,4,9,11$ & Both \\
\hline
\end{tabular}

AF, ameloblastic fibroma; AFD, ameloblastic fibrodentinoma; AFO, ameloblastic fibro-odontoma.

${ }^{a}$ Case 5 was manually microdissected into tumor and capsule.

http://erc.endocrinology-journals.org DOI: 10.1530/ERC-15-0094
(C) 2015 Society for Endocrinology Printed in Great Britain
Published by Bioscientifica Ltd 


\section{Analysis of H\&E staining of orofacial tissues from Prkar1a $^{+/-}$mice}

There were no abnormalities in any of the mice that were studied.

\section{Discussion}

There have been few investigations about the molecular pathogenesis of mixed odontogenic tumors because of the rarity of these lesions. The majority of studies have been based on IHC findings for diagnostic and prognostic biomarkers. After the identification of the first PRKAR1A mutations in humans with a variety of tumors, including myxomas (Kirschner et al. 2000a,b), PRKAR1A was shown to be involved in the pathogenesis of odontogenic myxomas both at the protein level and due to somatic mutations at the genetic level, as reported by Perdigão et al. (2005). The odontogenic myxoma is an odontogenic tumor that probably derived from the ectomesenchymal tissue during disruption ofodontogenesis (reviewed by Gomes et al. (2011)). Mixed odontogenic tumors share this ectomesenchymal origin as well as the participation of the odontogenic epithelium. Therefore, it is possible that these tumors share similarities with the odontogenic myxomas in their molecular pathogenesis.

Because we have previously shown PRKAR1A and PRKAR2A expression in tooth germs (de Sousa et al. 2014), we initially investigated their expression in mixed odontogenic tumors and AMs, which are the most common epithelial odontogenic tumors. We focused the present study on PRKAR1A, because it showed decreased mRNA levels in odontogenic tumors as compared with normal OM and because of the lack of PRKAR1A protein expression in some mixed odontogenic tumors. In addition, about half of these benign tumors showed a loss in PRKAR1A protein expression, mainly in their mesenchymal portion. AM samples, on the other hand, did not show a loss of PRKAR1A protein expression, so we therefore decided to further investigate PRKAR1A only in mixed odontogenic tumors. Cases with decreased protein expression had also undergone LOH at the PRKAR1A locus in the epithelial and/or mesenchymal component, which indicates that the LOH found in these tumors could be one reason for their loss of protein expression. Conversely, although one AFS exhibited $\mathrm{LOH}$ in two of the three informative markers in the epithelial component, the two cases of AFS showed moderate expression of PRKAR1A protein and did not reveal the presence of PRKAR1A mutations. Despite the small number of AFS samples, these findings indicate that
PRKAR1A alterations may play an important role in the pathogenesis of benign mixed odontogenic tumors. Another possibility is that, as previously proposed for adrenocortical adenomas (Bertherat et al. 2003), PRKAR1A down-regulation may be an early event in tumorigenesis, but it is quickly bypassed by other pathways that are more important for cell survival.

New missense and synonymous variants were also found in noncoding regions of PRKAR1A, such as the $5^{\prime}$-UTR region and introns in four benign mixed odontogenic tumors. Similarly to the missense mutation that was previously found in odontogenic myxomas (Perdigão et al. 2005), the missense mutation described in the present study occurred in the second cAMP binding domain. This may have effects on the interaction of this protein with its ligand, cAMP, which in turn affect its function. The effect of this substitution was predicted to be damaging in silico. The absence of $\mathrm{LOH}$ and the strong immunoexpression seen in both components of this sample indicate that either the substitution p.A268T did not cause a decrease in PRKAR1A protein expression that could be detected by IHC or that the mutation occurred only in a group of cells as a result of tumor heterogeneity. The missense mutation occurred in the somatic state in the ectomesenchymal part of an AFO case of one of the youngest patients in our series ( 6 years). The presence of a missense mutation in only the AFO case is interesting, seeing as there is ongoing debate in the literature about the true neoplastic nature of the AFO versus it being considered a developing odontoma (i.e., a hamartoma) (Buchner et al. 2013).

The synonymous sequence alterations that were also found in the somatic state are believed to be silent, because they do not alter the protein sequence. However, it has recently been proposed that such changes could affect mRNA stability and/or the timeliness of translation, the efficiency of splicing, and other factors that affect gene expression (Supek et al. 2014, Zheng et al. 2014). Moreover, there is increasing evidence that noncoding DNA changes may affect disease susceptibility (Castillo-Davis 2005). Some noncoding regions are recurrently mutated in cancer, especially regulatory regions at the $5^{\prime}$ ends of genes, such as promoters and $5^{\prime}$-UTRs (Weinhold et al. 2014). Although the significance of the $5^{\prime}$-UTR changes that we found remains unknown, the results of some studies have indicated that truncations or mutations in the $5^{\prime}$-UTR often lead to impaired protein synthesis, as demonstrated in the analysis of the 5'-UTR BRCA1 mRNA, which revealed that a $\mathrm{G} \rightarrow \mathrm{C}$ mutation close to the end of

Published by Bioscientifica Ltd. 
the $5^{\prime}$-UTR was associated with decreased BRCA1 gene expression (Signori et al. 2001).

Human tumors often display intratumor heterogeneity in histology, gene expression, and genotype (Michor \& Polyak 2010). An interesting finding in the present study is the molecular heterogeneity found when we compared the epithelial component and the mesenchymal cells of the tumors, which showed differences in $\mathrm{LOH}$ and the presence (or absence) of sequence changes. This could only be achieved by using laser microdissection, and it is the first evidence, to our knowledge, that these tumor parts are different not only histologically but also molecularly.

The Prkar1a ${ }^{+/-}$orofacial tissues that we examined did not demonstrate any odontogenic tumor formation and had normal odontogenesis without any disruption, even at a young age ( $1 \mathrm{month}$ ). The present results are consistent with the results of Almeida et al. (2010), who provided evidence that Prkar1a haploinsufficiency is a relatively weak tumorigenic signal that acts synergistically most of the time (Almeida et al. 2010). It should be noted that there is no question that PRKAR1A participates in the formation of orofacial structures, as has been demonstrated by knocking out both alleles of the Prkar1a gene in tyrosinase-expressing Cre 1 mice, which resulted in profound craniofacial abnormalities (Jones et al. 2010). To date, no patients with Carney complex have developed any orofacial tumors (other than schwannomas); however, skeletal tumors, albeit rare, are present.

In conclusion, the results of the present study indicated that PRKAR1A expression is decreased in benign mixed odontogenic tumors in association with somatic sequence alterations and LOH of the PRKAR1A gene. Although no abnormalities were found in Prkar1 ${ }^{+/-}$mice, these results indicate that PRKAR1A and perhaps other molecules of the cyclic AMP-dependent PKA pathway participate in the pathogenesis of these rare tumors.

\section{Declaration of interest}

The authors declare that there is no conflict of interest that could be perceived as prejudicing the impartiality of the research reported.

\section{Funding}

This study was supported in part by the intramural research program (IRP) of NICHD, NIH, and by the following Brazilian funding agencies: Coordination for the Improvement of Higher Education Personnel (CAPES), Fundação de Amparo à Pesquisa do Estado de Minas Gerais (FAPEMIG), and the National Council for Scientific and Technological Development (CNPq), Brazil. R S Gomez and C C Gomes are research fellows at CNPq, and M G Diniz is a research fellow at CAPES.

\section{Acknowledgements}

The authors acknowledge the Centro de Aquisição e Processamento de Imagens (CAPI- ICB/UFMG) for the LMD technical support and Dr Adriano Loyola for the donation of two tumor samples for the study.

\section{References}

Almeida MQ, Muchow M, Boikos S, Bauer AJ, Griffin KJ, Tsang KM, Cheadle C, Watkins T, Wen F, Starost MF et al. 2010 Mouse Prkar1a haploinsufficiency leads to an increase in tumors in the $\operatorname{Tr} 553^{+/-}$or $R b 1^{+/-}$ backgrounds and chemically induced skin papillomas by dysregulation of the cell cycle and Wnt signaling. Human Molecular Genetics 19 1387-1398. (doi:10.1093/hmg/ddq014)

Barnes L, Eveson JW, Reichart P \& Sidransky D eds 2005 Odontogenic tumours. In Pathology and Genetics of Head and Neck Tumours, WHO Classification of Tumours, vol 9, ch 6, pp 283-318. Eds L Barnes, JW Eveson, P Reichart \& D Sidransky. Lyon: IARC Press.

Barreto DC, Gomez RS, Bale AE, Boson WL \& De Marco L 2000 PTCH gene mutations in odontogenic keratocysts. Journal of Dental Research 79 1418-1422. (doi:10.1177/00220345000790061101)

Bernardes VF, Gomes CC \& Gomez RS 2012 Molecular investigation of ameloblastic fibroma: how far have we gone? Head \& Neck Oncology 445.

Bertherat J, Groussin L, Sandrini F, Matyakhina L, Bei T, Stergiopoulos S, Papageorgiou T, Bourdeau I, Kirschner LS, Vincent-Dejean C et al. 2003 Molecular and functional analysis of PRKAR1A and its locus (17q22-24) in sporadic adrenocortical tumors: $17 \mathrm{q}$ losses, somatic mutations, and protein kinase A expression and activity. Cancer Research 63 5308-5319.

Buchner A, Kaffe I \& Vered M 2013 Clinical and radiological profile of ameloblastic fibro-odontoma: an update on an uncommon odontogenic tumor based on a critical analysis of 114 cases. Head and Neck Pathology 7 54-63. (doi:10.1007/s12105-012-0397-9)

Castillo-Davis CI 2005 The evolution of noncoding DNA: how much junk, how much func? Trends in Genetics 21 533-536. (doi:10.1007/s12105012-0397-9)

Farias LC, Gomes CC, Brito JA, Galvão CF, Diniz MG, de Castro WH, Bernardes V de F, De Marco LA \& Gomez RS 2012 Loss of heterozygosity of the PTCH gene in ameloblastoma. Human Pathology 43 1229-1233. (doi:10.1016/j.humpath.2011.08.026)

Galvão CF, Gomes CC, Diniz MG, Vargas PA, de Paula AM, MosquedaTaylor A, Loyola AM \& Gomez RS 2012 Loss of heterozygosity (LOH) in tumor suppressor genes in benign and malignant mixed odontogenic tumors. Journal of Oral Pathology \& Medicine 41 389-393.

Gomes CC \& Gomez RS 2011 PTCH1 gene inactivation is not a keratocystic odontogenic tumor exclusive alteration. Oral Oncology 47 226-227. (doi:10.1016/j.oraloncology.2010.12.004)

Gomes CC, Diniz MG, Duarte AP, Bernardes VF \& Gomez RS 2011 Molecular review of odontogenic myxoma. Oral Oncology 47 325-328. (doi:10.1016/j.oraloncology.2011.03.006)

Jones GN, Pringle DR, Yin Z, Carlton MM, Powell KA, Weinstein MB, Toribio RE, La Perle KM \& Kirschner LS 2010 Neural crest-specific loss of Prkar1a causes perinatal lethality resulting from defects in intramembranous ossification. Molecular Endocrinology 24 1559-1568. (doi:10.1210/me.2009-0439)

Kirschner LS, Carney JA, Pack SD, Taymans SE, Giatzakis C, Cho YS, Cho-Chung YS \& Stratakis CA $2000 a$ Mutations of the gene encoding the protein kinase A type I- $\alpha$ regulatory subunit in patients with the Carney complex. Nature Genetics 26 89-92. (doi:10.1038/79238)

Kirschner LS, Sandrini F, Monbo J, Lip JP, Carney JA \& Stratakis CA $2000 b$ Genetic heterogeneity and spectrum of mutations of the PRKAR1A gene in patients with the Carney complex. Human Molecular Genetics 9 3037-3046. (doi:10.1093/hmg/9.20.3037)

Kirschner LS, Kusewitt DF, Matyakhina L, Towns WH II, Carney JA, Westphal H \& Stratakis CA 2005 A mouse model for the Carney

Published by Bioscientifica Ltd. 
complex tumor syndrome develops neoplasia in cyclic AMP-responsive tissues. Cancer Research 65 4506-4514. (doi:10.1158/0008-5472. CAN-05-0580)

Livak KJ \& Schmittgen TD 2001 Analysis of relative gene expression data using real-time quantitative PCR and the $2^{-\Delta \Delta C T}$ method. Methods 25 402-408. (doi:10.1006/meth.2001.1262)

Maleszewski JJ, Larsen BT, Kip NS, Castonguay MC, Edwards WD, Carney JA \& Kipp BR 2014 PRKAR1A in the development of cardiac myxoma: a study of 110 cases including isolated and syndromic tumors. American Journal of Surgical Pathology 38 1079-1087. (doi:10.1097/PAS. 0000000000000202)

Michor F \& Polyak K 2010 The origins and implications of intratumor heterogeneity. Cancer Prevention Research 3 1361-1364. (doi:10.1158/ 1940-6207.CAPR-10-0234)

Perdigão PF, Gomez RS, Pimenta FJ \& De Marco L 2004 Ameloblastin gene $(A M B N)$ mutations associated with epithelial odontogenic tumors. Oral Oncology 40 841-846. (doi:10.1016/j.oraloncology.2004.03.004)

Perdigão PF, Stergiopoulos SG, De Marco LG, Matyakhina L, Boikos SA, Gomez RS, Pimenta FJ \& Stratakis CA 2005 Molecular and immunohistochemical investigation of protein kinase A regulatory subunit type 1A (PRKAR1A) in odontogenic myxomas. Genes, Chromosomes \& Cancer 44 204-211. (doi:10.1002/gcc.20232)

Philipsen HP, Reichart PA \& Praetorius F 1997 Mixed odontogenic tumors and odontomas. Considerations on inter-relationship. Review of the literature and presentation of 134 new cases of odontomas. Oral Oncology 33 86-99. (doi:10.1016/S09641955(96)00067-X)
Praetorius F 2009 Odontogenic tumors. In Surgical Pathology of the Head and Neck, pp 1240-1247. Ed L Barnes., 3rd edn New York, NY: Informa Healthcare USA.

Robinson-White AJ, Leitner WW, Aleem E, Kaldis P, Bossis I \& Stratakis CA 2006 PRKAR1A inactivation leads to increased proliferation and decreased apoptosis in human B lymphocytes. Cancer Research 66 10603-10612. (doi:10.1158/0008-5472.CAN-06-2200)

Signori E, Bagni C, Papa S, Primerano B, Rinaldi M, Amaldi F \& Fazio VM 2001 A somatic mutation in the $5^{\prime}$-UTR of BRCA1 gene in sporadic breast cancer causes down-modulation of translation efficiency. Oncogene 20 4596-4600. (doi:10.1038/sj.onc.1204620)

de Sousa SF, Kawasaki K, Kawasaki M, Volponi AA, Gomez RS, Gomes CC, Sharpe PT \& Ohazama A 2014 PKA regulatory subunit expression in tooth development. Gene Expression Patterns 15 46-51. (doi:10.1016/ j.gep.2014.04.002)

Strokes A, Drozdov I, Guerra E, Ouzounis CA, Warnakulasuriya S, Gleeson MJ McGurk M, Tavassoli M \& Odell EW 2011 Copy number and loss of heterozygosity detected by SNP array of formalin-fixed tissues using whole-genome amplification. PLOS ONE 6 e24503. (doi:10.1371/ journal.pone.0024503)

Supek F, Miñana B, Valcárcel J, Galbadón T \& Lehner B 2014 Synonymous mutations frequently act as driver mutations in human cancers. Cell 156 1324-1335. (doi:10.1016/j.cell.2014.01.051)

Weinhold N, Jacobsen A, Schultz N, Sander C \& Lee W 2014 Genome-wide analysis of noncoding regulatory mutations in cancer. Nature Genetics 46 1160-1165. (doi:10.1038/ng.3101)

Zheng S, Kim H \& Verhaak RG 2014 Silent mutations make some noise. Cell 156 1129-1131. (doi:10.1016/j.cell.2014.02.037)

Received in final form 23 March 2015

Accepted 30 March 2015

Made available online as an Accepted Preprint

13 April 2015
(C) 2015 Society for Endocrinology Printed in Great Britain 